\title{
DNA Major Groove Binding
}

National Cancer Institute

\section{Source}

National Cancer Institute. DNA Major Groove Binding. NCI Thesaurus. Code C40493.

DNA Major Groove Binding involves temporary non-covalent interaction through

intermolecular physical forces of attraction by a protein or low molecular weight lig and with double-stranded DNA in the larger (major) groove formed between successive turns by the double helical structure, in either a sequence dependent or independent fashion. 